\title{
An Xp11.2 translocation renal cell carcinoma with SMARCB1 (INI1) inactivation in adult end-stage renal disease: a case report
}

\author{
Lu Yu ${ }^{1,2}$, Jun $\mathrm{Li}^{1,2}$, Sanpeng $X \mathrm{u}^{1}$, Mariajose Navia Miranda ${ }^{1}$, Guoping Wang ${ }^{1,2}$ and Yaqi Duan ${ }^{1,2^{*}}$
}

\begin{abstract}
Background: Xp11.2 translocation/transcription factor E3 (TFE3) rearrangement renal cell carcinoma (RCC) is a rare subtype of RCC with limited clinical and pathological data.

Case presentation: Here we present an unusual high-grade Xp11.2 translocation RCC with a rhabdoid feature and SMARCB1 (INI1) inactivation in a 40-year-old man with end-stage kidney disease. The histological examination of the dissected left renal tumor showed an organoid architecture of the eosinophilic or clear neoplastic cells with necrosis and high mitotic activity. In some areas, non-adhesive tumor cells with eccentric nuclei were observed. Immunohistochemically (IHC), the tumor cells are positive for TFE3 and the renal tubular markers (PAX2 and PAX8), and completely negative for SMARCB1, an oncosuppressor protein. Break-apart florescence in situ hybridization and reverse transcription polymerase chain reaction confirmed TFE3 rearrangement on Xp11.2 and the presence of ASPSCR1-TFE3 fusion gene. DNA sequencing revealed a frameshift mutation in exon 4 of SMARCB1 gene.
\end{abstract}

Conclusion: It is important to recognize this rare RCC with both TFE3 rearrangement and SMARCB1 inactivation, as the prognosis and therapeutic strategies, particularly targeted therapies for such tumors, might be different.

Keywords: Renal cell carcinoma, Kidney, TFE3, Xp11.2, SMARCB1

\section{Background}

Renal cell carcinomas (RCCs) represent $90 \%$ of all malignancies of the kidney in adults and $<5 \%$ of the malignancies in children. WHO subdivision of this entity includes a group of neoplasms distinguished by chromosomal Xp11.2 translocations resulting in the fusion of the transcription factor E3 (TFE3) gene to one of the different partners, including ASPSCR1, PRCC, NonO $\left(\mathrm{p} 54^{\mathrm{nrb}}\right)$, PSF and CLTC [1], with the consecutive overexpression of the chimeric protein TFE3. The diagnosis of Xp11.2 translocation RCC is not defined based on morphological features, but rather the

\footnotetext{
* Correspondence: yqduan@hust.edu.cn

${ }^{1}$ Institute of Pathology, Tongji Hospital, Tongji Medical College, Huazhong University of Science and Technology, Wuhan 430030, People's Republic of China

${ }^{2}$ Department of Pathology, School of Basic Medical Science, Huazhong University of Science and Technology, Wuhan 430030, People's Republic of China
}

genetic identification of Xp11.2 translocation using

The gene $h S N F / I N I 1 / S M A R C B 1 / B A F 47$ is a putative tumor suppressor gene expressed in all normal cells. The inactivation of SMARCB1 has been observed in malignant rhabdoid tumors (MRT), childhood atypical teratoid/rhabdoid tumor (AT/RT) of the central nervous system (CNS), epithelioid sarcoma [2], subsets of collecting duct carcinoma [3] and epithelioid malignant peripheral nerve sheath tumor (MPNST) [4], renal medullary carcinoma [5] and undifferentiated pediatric sarcomas [6], etc. The loss of SMARCB1 nuclear renal MRT and AT/RT [7].

Here we presented a high-grade malignant renal cell cancer with TFE3 translocation and SMARCB1 inactivation in an end-stage kidney. 


\section{Case presentation}

A 40-year-old man with chronic renal failure and undergoing long-term hemodialysis (7 years) was admitted to the hospital as a result of chronic flank pain. Subsequent Magnetic Resonance Image (MRI) of the abdomen revealed an approximately $12 \mathrm{~cm} \times 6 \mathrm{~cm} \times 5 \mathrm{~cm}$ solid mass in the right kidney with calcification, a cystic lesion in the left kidney and enlargement of the retroperitoneal lymph nodes (Fig. 1). Radical nephrectomy of the right kidney was performed.

Grossly, the renal parenchyma was almost replaced with a grayish tan and fleshy tumor with focal necrosis, hemorrhage and calcification. The tumor invaded the renal pelvis, calyces and the hilar area of the kidney and extended to the capsule.

The tissues were fixed in $10 \%$ buffered formalin solution, embedded in paraffin block, $4 \mu \mathrm{m}$ thick sections were obtained and subsequently stained with hematoxylineosin. Under the microscope the tumor cells were arranged in an organoid pattern with a well-defined cell border and eosinophilic voluminous cytoplasm. The nucleus was enlarged and possessed vesicular chromatin with apparent nucleoli, indicating a high nuclear grade. In some areas, rhabdoid cells were observed. These rhabdoid cells were non-adhesive and showed eccentric nuclei and intracytoplasmic inclusions of eosinophilic hyaline globules (Fig. 2a, b). The high malignancy was indicated by high mitotic activities (Ki 67 index approximately $40 \%$ ) and increased necrosis in the lesion. The tumor invaded to the adipose tissue of the renal hilum.

Next, immunohistochemistry was performed on the sections to assess tumor classification using an avidinbiotin peroxidase technique with hematoxylin counterstaining. The antibodies used in the present study included anti-vimentin (V9, 1:400, Dako), anti-pan-CK (AE1/AE3, 1:200, DAKO), anti-PAX2 (EP3251, 1:1000, Abcam), anti-PAX8 (ZR-1, 1:100, Abcam), anti-HMB45 (HMB45 + 50, 1:100, DAKO), anti-MelanA (A103, 1:100,
DAKO), anti-MyoD1 (58A, 1:100, Santa Cruz), antiMyogenin (F5D, 1:50, Santa Cruz), anti-TFE3 (H-300, 1:100, Santa Cruz), and anti-SMARCB1 (H-300, 1:100, Santa Cruz). As demonstrated in Fig. 2g-j, vimentin and pan-CK were both positive in all tumor cells, with more prominent perinuclear and intense cytoplasmic staining in rhabdoid cells (Fig. 2h, j). The neoplastic cells showed positive staining for PAX2 and PAX8, indicating renal tubular differentiation, and negative staining for CD10, HMB45, MelanA, myogenin, myoD1, desmin, CD31 and CD34. Interestingly, we observed that all tumor cells showed the complete loss of nuclear staining for SMARCB1 in both the organoid and non-adhesive areas with rhabdoid cells compared with the ubiquitous expression of SMARCB1 in surrounding normal tissue (Fig. 2e, f), indicating a renal malignant rhabdoid tumor. Surprisingly, the tumor cells demonstrated strong nuclear and weak cytoplasmic staining for TFE3 (Fig. 2c, d), a relatively specific IHC marker for Xp11.2 translocation RCC.

To determine the TFE3 rearrangement status, we performed FISH in paraffin-embedded material using a probe comprising 2 contigs flanking the TFE3 gene on Xp11.2 (ZytoLight SPEC TFE3). For males, one fuse signal reflects an intact TFE3 allele. Positivity was defined based on the separation of red and green signals via more than 2 signal diameters (split signals). We used a cutoff of $>10 \%$ of the tumor nuclei with any pattern of break-apart signals. In this case, approximately $15 \%$ of the tumor cells showed split signals, and a copy number gain of TFE3 gene was also observed in some tumor cells with or without translocation ( 3 to 5 signals per nucleus) (Fig. 3a). RNA was extracted from the formalinfixed and paraffin-embedded tumor samples using a FFPE DNA/RNA Kit (AmoyDx, Guangzhou, China), and subsequently reverse transcription-polymerase chain reaction (RT-PCR) was performed using the PrimeScript ${ }^{\mathrm{Tm}}$ Reagent kit (Takara, Dalian, Japan). The primers used to detect the
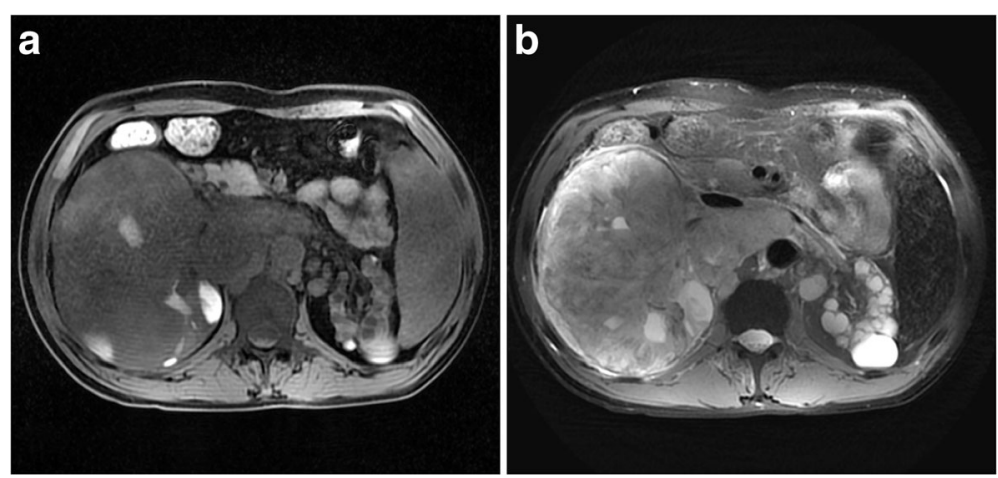

Fig. 1 Magnetic Resonance Image (MRI) of the abdomen in a 40-year-old man with Xp11.2 translocation RCC. a, b, Axial T2WI (a) and plain T1WI (b) showed a large, well-defined, irregular mass ( $T 2$, high-low heterogeneous signal intensity; $T 1$, iso-signal intensity) with patchy hemorrhage and necrosis in the mass and enlargement of abdominal lymph nodes 

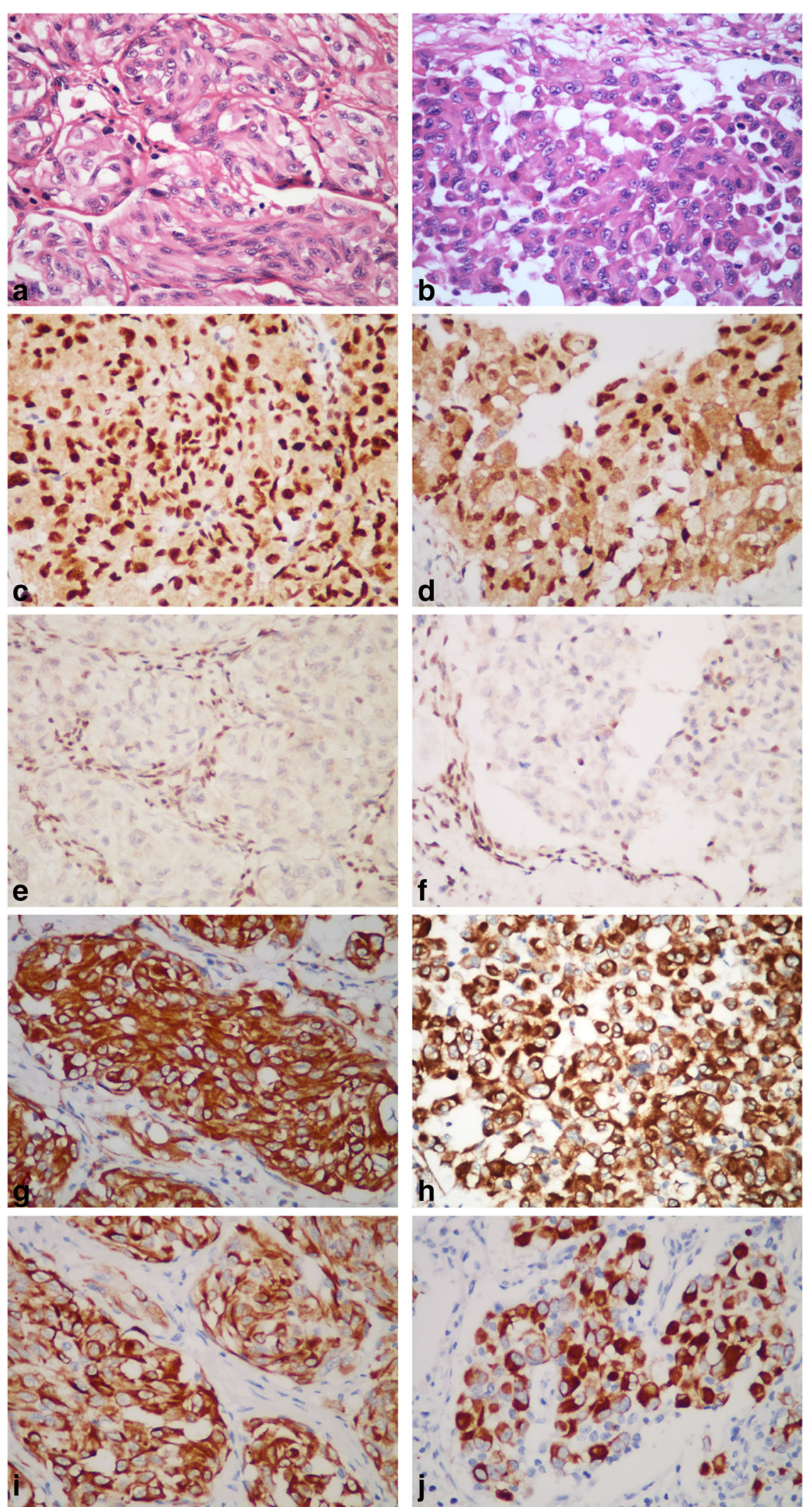

Fig. 2 (See legend on next page.) 
(See figure on previous page.)

Fig. 2 Histopathological and immunohistochemical features of the renal tumor. $\mathbf{a}$, b Nested eosinophilic tumor cells (a) and non-cohesive tumor cells (b) with abundant pink cytoplasm and eosinophilic intracytoplasmic inclusions (H\&E staining, 400X magnification). c, $\mathbf{d}$, e, $\mathbf{f}$ Neoplastic cells in both organoid and non-adhesive areas demonstrated strong nuclear staining of TFE3 (c, d) and negative staining of INI1 (e, f) (400X magnification). $\mathbf{g}, \mathbf{h}, \mathbf{i}, \mathbf{j}$ All tumor cells showed strong positive staining for vimentin $(\mathbf{g}, \mathbf{h})$ and pan-CK $(\mathbf{i}, \mathbf{j})$ with prominent perinuclear and cytoplasmic staining in non-adhesive area (400X magnification)

$\mathbf{a}$
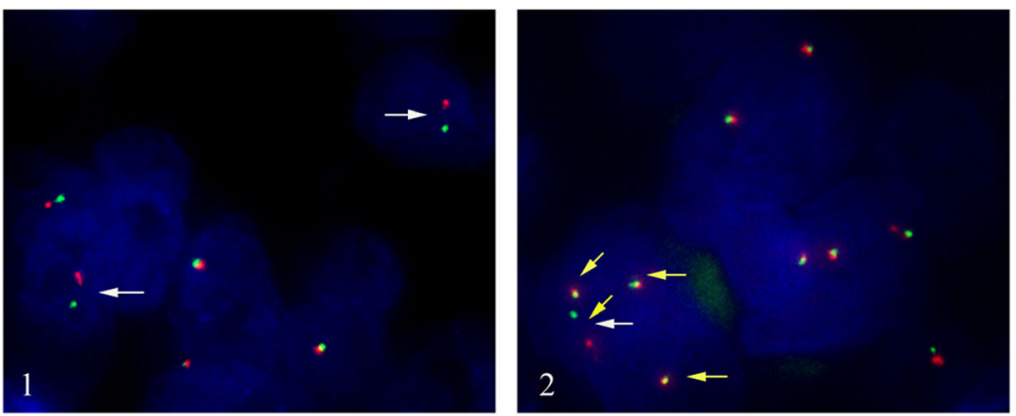

b
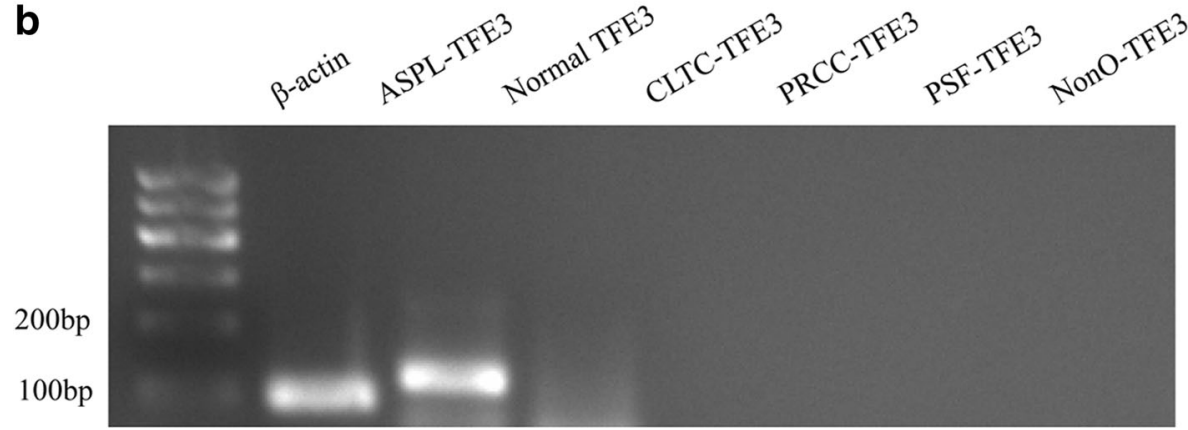

C

Ins

G T G G T G G $\stackrel{140}{A} \mathrm{G} C \mathrm{~T} G \mathrm{~T} T \stackrel{\downarrow}{\mathrm{T}} \mathrm{G} G \stackrel{150}{\mathrm{G}} \mathrm{CA}$ A G G T G G G $\mathrm{T} A$

G T G G T G G $\stackrel{140}{A} \mathrm{G} C \mathrm{~T} G \mathrm{~T} T \stackrel{\mathrm{T}}{\mathrm{G}} \mathrm{G} \stackrel{150}{\mathrm{G}} \mathrm{CA}$ A G G T G G G $\mathrm{T}$ A

G T G G T G G G A G C T G T T $\stackrel{140}{T} G \stackrel{150}{\mathrm{G}} \mathrm{CA}$ A G G T G G G $\mathrm{T}$ A

G T G G T G G A C T G T G G G A G G T G G G A C

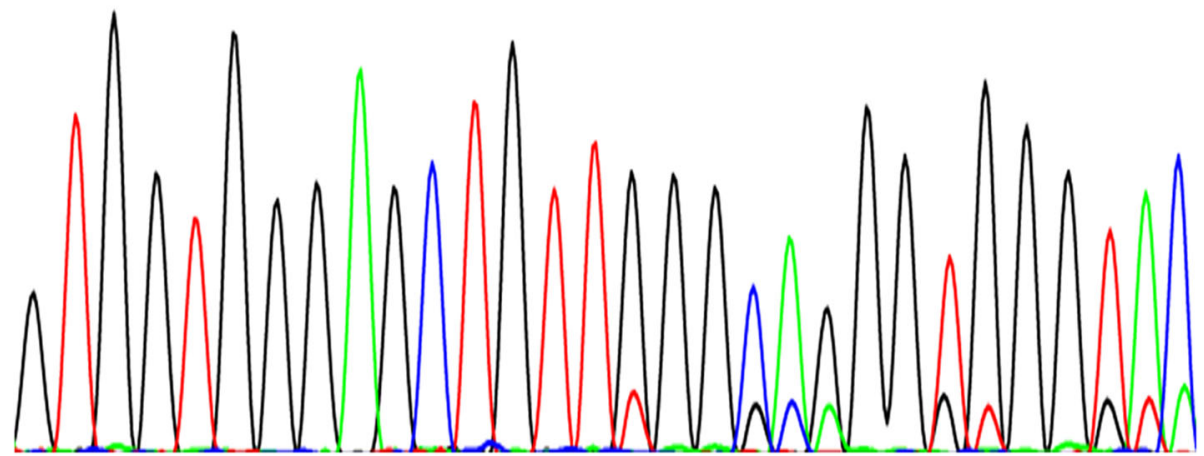

Fig. 3 Molecular genetic analyses for the renal tumor. a The TFE3 break-apart probe assay identified split signals (white arrow) and increased TFE3 copy numbers (yellow arrow, 4 signals in one nucleus including one split signal). b Reverse transcription polymerase chain reaction detected an ASPSCR1TFE3 fusion gene product. c Sequence analysis of SMARCB1 gene (exon 1-9) demonstrated c.147InsT in exon 4, causing a frameshift alteration 
gene fusion products of all translocation types (ASPSCR1-TFE3, PRCC-TFE3, PSF-TFE3, CLTC-TFE3 and Nono-TFE3) are listed in Table 1. A band for the ASPSCR1-TFE3 fusion product is demonstrated in Fig. 3b. Thus, an ASPSCR1-TFE3 translocation RCC was considered.

In addition to the TFE3 rearrangement, the tumor cells showed the complete loss of SMARCB1 expression. Subsequent DNA sequencing to determine the genetic alteration of SMARCB1 (exon 1-9) revealed a frameshift mutation in exon 4 of SMARCB1 (Fig. 3c).

Taken together, a diagnosis of Xp11.2 translocation RCC was preferred. Considering the loss of SMARCB1 expression observed herein, this case presents a unique Xp11.2 translocation RCC with SMARCB1 inactivation.

This patient died 6 months after the surgery.

\section{Discussion}

Xp11 translocation RCC commonly demonstrates clear cells with papillary, cystic, tubular, and organoid architecture, and psammoma bodies are common in the lesions. Occasionally pleomorphic giant cells and sarcomatoid differentiation are observed. The diagnosis of Xp11 translocation RCC depends on the detection of an Xp11 translocation using a TFE3 break-apart FISH assay performed on paraffin-embedded tissue [1]. Strong nuclear TFE3 immunoreactivity, demonstrating the TFE3 fusion protein resulting from an $\mathrm{Xp} 11$ translocation, is helpful for the diagnosis. However, the TFE3 IHC assay is technically challenging, reflecting suboptimal fixation and detection methods, and occasionally TFE3 amplification could serve as additional underlying genomic alterations for TFE3 overexpression $[1,8]$. In the present

Table 1 Primers used to detect the gene fusion product of Xp11.2 translocation RCC

\begin{tabular}{ll}
\hline Name & Primer (5' $\rightarrow$ 3') \\
\hline$\beta$-actin(F) & ATCACCATTGGCAATGAGCG \\
$\beta$-actin(R) & TTGAAGGTAGTTTCGTGGAT \\
PSF-TFE3(F) & TGGTGGTGGCATAGGTTATG \\
PSF-TFE3(R) & CGTTTGATGTTGGCAGCTC \\
NonO(p54nrb)-TFE3(F) & GAGAAACTAGACACAGCAAC \\
NonO(p54nrb)-TFE3(R) & CTTCTTCTGCCGTTCCTC \\
PRCC-TFE3(F) & CCAAGCCAAAGAAGAGGA \\
PRCC-TFE3(R) & AGTGTGGTGGACAGGTACTG \\
CLTC-TFE3(F) & AGTCGCGTTGTTGGAAAGTATTGTG \\
CLTC-TFE3(R) & CAAAAGGGCCTTGGCTCGGTC \\
ASPSCR1-TFE3(F) & AAAGAAGTCCAAGTCGGGCCA \\
ASPSCR1-TFE3(R) & CGTTTGATGTTGGGCAGCTCA \\
Normal TFE3(exon3) (F) & CCCGCAAGTGCCCAGCCACTG \\
Normal TFE3(exon4) (R) & CAGTTCCTTGATCCTGTCG \\
\hline
\end{tabular}

case, the tumor cells with organoid architecture separated by fibrovascular network showed strong TFE3 nuclear staining, a split signal of TFE3 genes, and ASPSCR1-TFE3 fusion PCR products, suggesting Xp11.2 translocation RCC. An increasing number of TFE3 rearrangement-associated tumors, which might morphologically overlap with RCC, including TFE3 rearrangementassociated perivascular epithelioid cell tumors (PEComas) and melanotic Xp11.2 translocation cancer $[9,10]$, have recently been reported. These tumors can be excluded by immunohistochemistry, as PEComas and melanotic Xp11.2 translocation cancer are negative for renal cell markers (CD10, PAX2 and PAX8) and positive for MelanA and HMB45.

Although a higher percentage of Xp11.2 translocation $\mathrm{RCC}$ has been observed in children, adult Xp11.2 translocation RCC is overall more common, reflecting the increased population of adult RCC patients. In adults, the occurrence of Xp11.2 translocation RCC has been correlated with chemotherapy, as approximately $15 \%$ of patients had a history of chemotherapy exposure [1]. The patient described herein had chronic renal failure and received 7 years of hemodialysis. The outcome data for the clinical behavior of adult Xp11.2 translocation RCC remains limited, although adult patients might present a worse prognosis and have a mean survival of up to 2 years when presenting metastases, in contrast to a favorable short-term prognosis and mean survival of 6.3 years in pediatric patients [1]. For adult Xp11.2 translocation RCC, ASPSCR1-TFE3 RCC is more likely to present at an advanced stage compared with PRCCTFE3 RCC. In addition to TFE3 rearrangement, MacherGoeppinger $\mathrm{S}$ et al. provided evidence that increased TFE3 expression resulting from gene amplification or epigenetic alterations was associated with unfavorable clinicopathological features, such as higher grade, the presence of metastatic disease and advanced tumor stage [8]. This case clearly correlated the presence of metastatic disease with the microscopic appearance of TFE3 overexpression resulted from TFE3 rearrangement and copy number gain. The optimal therapy for the Xp11.2 translocation RCC remains unknown, as clinical studies in a large population of patients remain absent. The current treatment for Xp11.2 RCC generally follows the guidelines of conventional RCC. Therapies targeting vascular endothelial growth factor receptor, C-met or mTOR in Xp11.2 RCC are not yet clear, and additional clinical studies are needed $[1,11]$.

In addition to cells rich in eosinophilic or clear cytoplasm and arranged in an organoid pattern, some of the tumor cells showed a non-cohesive rhabdoid feature. Accumulating data suggest that tumors with rhabdoid features contain a clinicopathological spectrum of neoplasms with a highly variable rhabdoid cell component, 
with or without the loss of nuclear SMARCB1 expression. Tumors lacking the biallelic inactivation of the SMARCB1 gene may develop rhabdoid morphologies. In the kidneys, the morphological rhabdoid differentiation of the primary tumor is typically associated with clear cell RCC and occasionally observed in papillary and chromophobe RCC, collecting duct carcinoma and acquired cystic disease associated RCC, representing a dedifferentiated status that indicates severe cytological anaplasia, a higher stage and a more aggressive biological behavior [1]. SMARCB1-deficient neoplasms might exhibit variable rhabdoid cell components. SMARCB1 deficiency was first observed in renal malignant rhabdoid tumors and childhood CNS AT/RTs. Subsequently the loss of nuclear SMARCB1 expression has been identified in renal medullary carcinoma, epithelioid sarcoma, a subset of collecting duct carcinoma and epithelioid malignant peripheral nerve sheath tumor (MPNST) and rare rhabdoid variants of carcinoma from digestive system and sinonasal tract [2-4, 12-14]. Apparently loss of SMARCB1 has been found in diverse tumors, which have not much in common except the loss of SMARCB1. The diagnosis of those tumors is still mainly based on the phenotype and the clinicopathologic features. Abbas Agaimy proposed that SMARCB1 deficient neoplasms were possibly derived from different progenitor cell subsets with different differentiation commitments in the different organs, and thus probably were genetic subtypes of tumors with certain differentiation [15]. Therefore, a diagnosis of Xp11.2 translocation RCC with SMARCB1 inactivation is proposed in this double hit tumor concerning the histopathological features and TFE3 rearrangement. Such diagnosis doesn't exclude the possibility that the INI1 loss is the primary event in this tumor.

Most tumors showing the loss of SMARCB1 expression are associated with aggressive biology and poor clinical outcomes. Although the functional role of SMARCB1 is not completely understood, the loss of SMARCB1 may cause cell cycle progression, and thus this gene might be involved in tumorigenesis via the dysregulation of cell cycle relevant molecules, including p16, Aurora A and cyclin D1, or the induction of DNA repair defects and genomic instability [16]. Concerning the absence of SMARCB1 in different types of tumors, such as epithelioid sarcomas and renal medullary carcinomas, the loss of SMARCB1 expression may be a biological event of uncertain prognostic significance. It is difficult to draw conclusions on the correlation between the loss of SMARCB1 expression and prognosis in the absence of larger studies. A recent finding on targeted therapy revealed that drugs inhibiting cyclin D1 and/or CDKs, such as fenretinide and flavopiridol, are effective in inhibiting rhabdoid tumor growth, correlated with the down-modulation of cyclin D1 [17].

\section{Conclusion}

In summary, both Xp11.2-associated translocation and the loss of SMARCB1 expression in renal cell carcinoma are rare and have not previously been reported. Highgrade Xp11.2 RCC with SMARCB1 inactivation is an extraordinary case, prompting the necessity to elucidate the clinical prognosis and develop therapeutic strategies.

\begin{abstract}
Abbreviations
AT/RT: Atypical teratoid/rhabdoid tumor; CNS: Central nervous system; FISH: Florescent in situ hybridization; IHC: Immunohistochemistry: MPNST: Epithelioid malignant peripheral nerve sheath tumor; MRI: Magnetic resonance image; MRT: Malignant rhabdoid tumors; PEComas: Perivascular epithelioid cell tumors; RCC: Renal cell carcinoma; RT-PCR: Reverse transcriptionpolymerase chain reaction; TFE3: Translocation/transcription factor E3
\end{abstract}

\section{Acknowledgment}

We thank the patient, who requested anonymity, for agreeing to our report and for providing a detailed medical history.

\section{Funding}

This project was financially supported by a grant from the Natural Science Foundation of China (31271040).

\section{Availability of data and materials}

The dataset supporting the conclusions of this article is included within the article.

\section{Authors' contributions}

LY carried out the molecular genetic studies, participated in the sequence alignment and drafted the manuscript. JL contributed to data collection for Fig. 3a (FISH) and Fig. 3b (RT-PCR), and helped with paper writing related with FISH and PCR experiments. SX carried out the immunoassays. MNM participated the revision of the manuscript and performed the statistical analysis. GW contributed to concept, approved the final version of the manuscript. YD conceived of the study, and participated in its design and made up the manuscript. All authors read and approved the final manuscript.

\section{Competing interests}

The authors declare that they have no competing interests.

\section{Consent for publication}

Written informed consent was obtained from the patient for the publication of this case report and any accompanying images. A copy of the written consent is available for review by the Editor-in-Chief of this journal.

\section{Ethics approval and consent to participate}

The Ethics Committee of Tongji Hospital, Tongji Medical College, Huazhong University of Science and Technology, Wuhan, China approved the following study, and written informed consent was obtained from the patient.

Received: 22 May 2016 Accepted: 8 October 2016

Published online: 12 October 2016

References

1. Srigley JR, Delahunt B, Eble JN, Egevad L, Epstein JI, Grignon D, et al. The International Society of Urological Pathology (ISUP) Vancouver classification of renal neoplasia. Am J Surg Pathol. 2013;37:1469-89.

2. Hornick JL, Dal Cin P, Fletcher CD. Loss of INI1 expression is characteristic of both conventional and proximal-type epithelioid sarcoma. Am J Surg Pathol. 2009:33:542-50.

3. Elwood H, Chaux A, Schultz L, Illei PB, Baydar DE, Billis A, et al. Immunohistochemical analysis of SMARCB1/INI-1 expression in collecting duct carcinoma. Urology. 2011;78:474. e1-5.

4. Jo WY, Fletcher CD. Epithelioid malignant peripheral nerve sheath tumor: clinicopathologic analysis of 63 cases. Am J Surg Pathol. 2015;39:673-82.

5. Cheng JX, Tretiakova M, Gong C, Mandal S, Krausz T, Taxy JB. Renal medullary carcinoma: rhabdoid features and the absence of INI1 expression as markers of aggressive behavior. Mod Pathol. 2008;21:647-52. 
6. Kreiger PA, Judkins AR, Russo PA, Biegel JA, Lestini BJ, Assanasen C, et al. Loss of INI1 expression defines a unique subset of pediatric undifferentiated soft tissue sarcomas. Mod Pathol. 2009;22:142-50.

7. Sigauke E, Rakheja D, Maddox DL, Hladik CL, White $\mathrm{CL}$, Timmons $\mathrm{CF}$, et al. Absence of expression of SMARCB1/INI1 in malignant rhabdoid tumors of the central nervous system, kidneys and soft tissue: an immunohistochemical study with implications for diagnosis. Mod Pathol. 2006:19:717-25.

8. Macher-Goeppinger S, Roth W, Wagener N, Hohenfellner M, Penzel R Haferkamp A, et al. Molecular heterogeneity of TFE3 activation in renal cell carcinomas. Mod Pathol. 2012;25:308-15.

9. Rao Q, Shen Q, Xia QY, Wang ZY, Liu B, Shi SS, et al. PSF/SFPQ is a very common gene fusion partner in TFE3 rearrangement-associated perivascular epithelioid cell tumors (PEComas) and melanotic Xp11 translocation renal cancers: clinicopathologic, immunohistochemical, and molecular characteristics suggesting classification as a distinct entity. Am J Surg Pathol. 2015;39:1181-96.

10. Argani P, Aulmann S, Karanjawala Z, Fraser RB, Ladanyi M, Rodriguez MM. Melanotic Xp11 translocation renal cancers: a distinctive neoplasm with overlapping features of PEComa, carcinoma, and melanoma. Am J Surg Pathol. 2009;33:609-19.

11. Malouf GG, Camparo P, Oudard S, Schleiermacher G, Theodore C, Rustine A, et al. Targeted agents in metastatic Xp11 translocation/TFE3 gene fusion renal cell carcinoma (RCC): a report from the Juvenile RCC Network. Ann Oncol. 2010;21:1834-8.

12. Calderaro J, Moroch J, Pierron G, Pedeutour F, Grison C, Maille P, et al. SMARCB1/INI1 inactivation in renal medullary carcinoma. Histopathology. 2012;61:428-35.

13. Pancione M, Di Blasi A, Sabatino L, Fucci A, Dalena AM, Palombi N, et al. A novel case of rhabdoid colon carcinoma associated with a positive CpG island methylator phenotype and BRAF mutation. Hum Pathol. 2011;42:1047-52.

14. Agaimy A, Koch M, Lell M, Semrau S, Dudek W, Wachter DL, et al. SMARCB1(INI1)-deficient sinonasal basaloid carcinoma: a novel member of the expanding family of SMARCB1-deficient neoplasms. Am J Surg Pathol. 2014;38:1274-81.

15. Agaimy A. The expanding family of SMARCB1(INI1)-deficient neoplasia: implications of phenotypic, biological, and molecular heterogeneity. Adv Anat Pathol. 2014;21:394-410.

16. Roberts CW, Biegel JA. The role of SMARCB1/INI1 in development of rhabdoid tumor. Cancer Biol Ther. 2009;8:412-6.

17. Smith ME, Cimica V, Chinni S, Jana S, Koba W, Yang Z, et al. Therapeutically targeting cyclin D1 in primary tumors arising from loss of Ini1. Proc Natl Acad Sci U S A. 2011;108:319-24.

\section{Submit your next manuscript to BioMed Central and we will help you at every step:}

- We accept pre-submission inquiries

- Our selector tool helps you to find the most relevant journal

- We provide round the clock customer support

- Convenient online submission

- Thorough peer review

- Inclusion in PubMed and all major indexing services

- Maximum visibility for your research

Submit your manuscript at www.biomedcentral.com/submit

CBiomed Central 